\title{
Epidemiological, Radio-Clinical and Histological Correlations of Breast Lesions ACR 3, ACR 4 and ACR 5 in the Ouagadougou' City
}

\author{
Massara Koné-Sigué1 ${ }^{*}$, Aïscha Madina Napon'1, Soré Moussa Zanga1, \\ Glwadys Cherlyn Béréhoudougou Kaboré1, Mathieu Kafando1, Pakisba Ali Ouédraogo², \\ Abel Yomboué Bamouni², Léonie Claudine Lougué-Sorgho ${ }^{1}$

\footnotetext{
${ }^{1}$ Department of Radiology, Pediatric University Hospital Center (CHUP) Charles De gaulles, Ouagadougou, Burkina Faso ${ }^{2}$ Department of Radiology, University Hospital Center (CHU) Yalgado Ouédraogo, Ouagadougou, Burkina Faso

Email: *massara_kone@yahoo.fr
}

How to cite this paper: Koné-Sigué, M., Napon, A.M., Zanga, S.M., Kaboré, G.C.B., Kafando, M., Ouédraogo, P.A., Bamouni, A.Y. and Lougué-Sorgho, L.C. (2020) Epidemiological, Radio-Clinical and Histological Correlations of Breast Lesions ACR 3, ACR 4 and ACR 5 in the Ouagadougou' City. Open Journal of Radiology, 10, 184-192. https://doi.org/10.4236/ojrad.2020.103018

Received: July 29, 2020

Accepted: September 25, 2020

Published: September 28, 2020

Copyright $\odot 2020$ by author(s) and Scientific Research Publishing Inc. This work is licensed under the Creative Commons Attribution International License (CC BY 4.0).

http://creativecommons.org/licenses/by/4.0/ (c) (i) Open Access

\begin{abstract}
Objective: To correlate the epidemiological and histological data of breast lesions ACR 3, ACR 4 and ACR 5 biopsied on ultrasound in the city of Ouagadougou. Materials and methods: This was a retrospective study of data for 02 years (2015-2017) in the city of Ouagadougou. With the approval of the Ethics Committee for Health Research, the study focused on the histological results and radiological reports of biopsied lesions. Results: A total of 150 patients files had been collected. Breast lesions classified as ACR 4 were $50 \%$, ACR $340 \%$ and ACR 5 10\%. The risk factors were related to the ACR class. Ultrasound was performed in all patients. The masses were the most common elementary lesions found in breast imaging. On histology 98 lesions were benign, 16 intermediate lesions and 36 malignant lesions. Fibroids were the most common benign lesions of the ACR 3 and ACR 4 classes. Invasive ductal carcinoma was the most common histologic type among malignant lesions in all ACR classes. Conclusion: Cancerous lesions are discovered late in our context. The ACR classification must always take into account discriminative elements.
\end{abstract}

\section{Keywords}

Breast Cancer, ACR, Echo-Mammography, Burkina Faso

\section{Introduction}

The leading cancer in women worldwide and the leading cause of death in industrialized countries, breast cancer has nowadays become a real public health 
problem. According to the World Health Organization, its incidence is gradually increasing in developing countries with a high mortality rate [1]. This mortality can be reduced thanks to a reliable and early diagnostic arsenal [2]. The advent of the Breast Imaging Reporting and Data System (BIRADS) classification from the American College of Radiology (ACR) helps a lot in diagnostic progress and remains effective if the different stakeholders in senology use the same terminologies [3]. The practical action to be taken with breast lesions being dependent on the ACR classification, it is necessary to have a consensus on it (see Annex). In our context where breast lesions are more and more discovered, what correlations exist between the ACR class found on ultrasound, mammography and histology of probably benign lesions, undetermined lesions and lesions suggestive of cancer?

\section{Materials and Methods}

This was a retrospective and descriptive study. It was carried out in the cytology and anatomy pathology departments as well as the medical imaging departments of the city of Ouagadougou in Burkina Faso. It concerned data for 02 years ranging from August 2015 to July 2017.

The study included consenting patients with ACR 3, 4 and 5 lesions detected by ultrasound and/or mammography and who had undergone a biopsy under ultrasound guidance and had their radio-histological reports.

The study was carried out after collecting the various radio-histological results using a questionnaire developed in French. Data collection histology from August 2015 to July 2017 concerning all specimens biopsied on ultrasound in the anatomo-pathology laboratories. The interview with the patient began after she read the information note and signed the consent certificate. Finally, the recovery of the radiological assessment followed to complete the collection sheet, which assessments were immediately returned to them.

The study protocol with reference to "version 1 of July 14, 2017" was submitted to the Ethics Committee for Health Research (CERS) for review. After deliberation $n^{\circ}$ 2017-9-140 of September 12, 2017, the CERS approved it by giving a favorable opinion for its implementation.

\section{Results}

A total of 150 records were collected from the lesions biopsied on ultrasound. Breast lesions classified ACR 4 were 75 (50\%) followed by ACR 360 (40\%) and ACR 515 (10\%).The distribution of breast lesions in ACR class is shown in Figure 1 .

The mean age of the patients was 36 years for ACR 3, 39 years for ACR 4 and 50 years for ACR 5 with respective age ranges of 16 to 63 years, 19 - 70 years and 35 - 70 years. The age group most affected by breast pathology was between 31 and 45 years old for all ACR classes and in particular ACR 5 class. The distribution of patients by age group by ACR is shown in Figure 2 . 


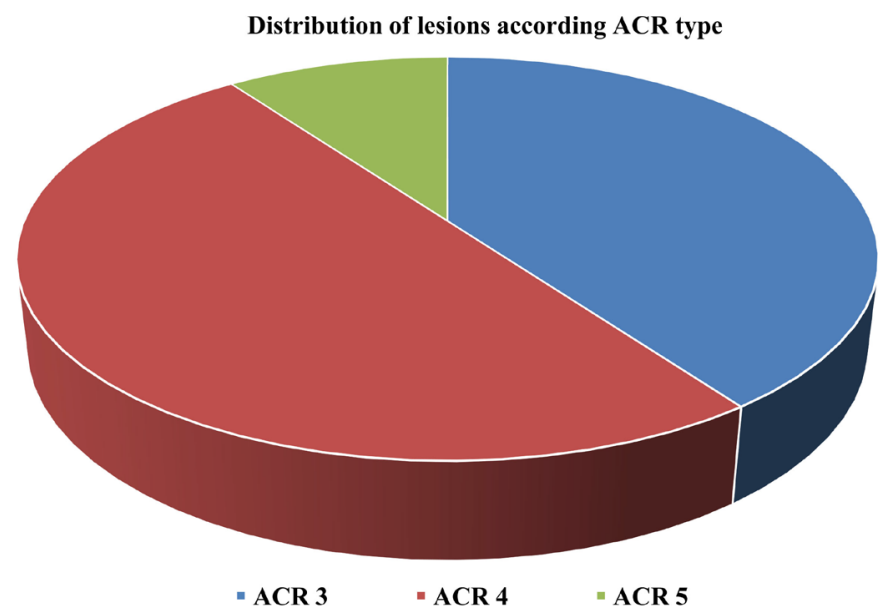

Figure 1. Distribution of breast lesions in ACR class.

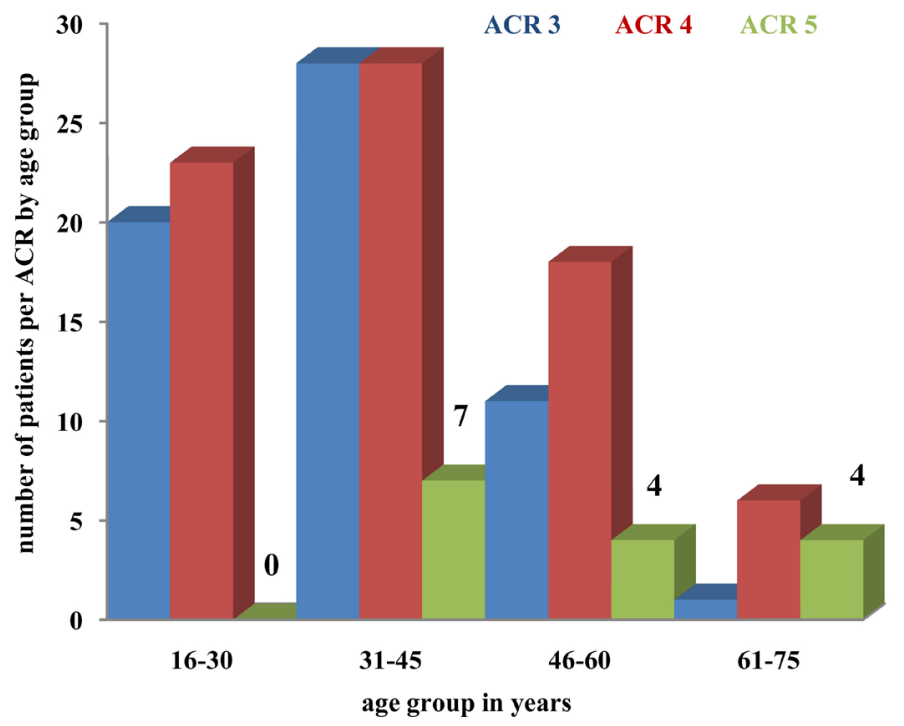

Figure 2. Distribution of patients by age group by ACR.

Eighteen (18) patients had a notion of a family history of breast cancer ( 9 of 1 st degree and 9 of 2 nd degree) either a rate of $12 \%$ and an increasing risk of cancer occurrence of $9 \%$ for ACR 3, 20\% for ACR 4 and 50\% for ACR 5.

Forty-seven patients were nulliparous and the risk of developing breast cancer increased from $0 \%$ for ACR 3, to 8.3\% for ACR 4 and 100\% for ACR 5.

Forty patients who did not exclusively breastfeed had an increasing risk of cancer development of $8 \%$ for ACR 3, $42 \%$ for ACR 4 and $87 \%$ for ACR 5.

The mean age of menopause of the patients was 46 years for ACR 3, 48 years for ACR 4 and 50 years for ACR 5 with respective age ranges of 40 to 42 years, 47 - 52 years and 48 - 55 years.

The presence of a clinical abnormality $(n=137)$ was the most frequent circumstance of discovery (CDD) of breast pathology dominated by palpation of nodules in 99 cases, a rate of $72 \%$. The distributions of these various discovery circumstances are shown in the tables (Tables 1-3): 
Table 1. Distribution of ACR 3 lesions according to the circumstance of discovery.

\begin{tabular}{cclcc}
\hline Circumstance of discovery & \multicolumn{1}{c}{ Types } & Effective & Frequency (\%) \\
\hline & - & Nodule or mass & 40 & 75 \\
Clinical anomaly & - & Mastodynia & 9 & 17 \\
$(\mathrm{n}=53)$ & - & Nodule + mastodynia & 2 & 3.7 \\
& - & Other & 2 & 3.7 \\
Tracking anomaly $(\mathrm{n}=3)$ & & - & 3 & - \\
Screening assessment $(\mathrm{n}=4)$ & & - & 4 & - \\
\hline
\end{tabular}

Table 2. Distribution of ACR 4 lesions according to the circumstance of discovery.

\begin{tabular}{cclcc}
\hline Circumstance of discovery & & \multicolumn{1}{c}{ Types } & Effective & Fréquency (\%) \\
\hline & - & Nodule or mass & 52 & 74 \\
& - & Mastodynia & 2 & 2.9 \\
Clinical anomaly & - & Nodule + mastodynia & 7 & 10 \\
$(\mathrm{n}=70)$ & - & Ulceration & 2 & 2.9 \\
& - & Other & 7 & 10 \\
Tracking anomaly $(\mathrm{n}=4)$ & & - & 4 & - \\
Screening assessment $(\mathrm{n}=1)$ & & - & 1 & - \\
\hline
\end{tabular}

Table 3. Distribution of ACR 5 lesions according to the circumstance of discovery.

\begin{tabular}{cclcc}
\hline Circumstance of discovery & \multicolumn{1}{c}{ Types } & Effective & Fréquency (\%) \\
\hline & - & Nodule or mass & 5 & 36 \\
Clinical anomaly & - & Inflammatory tuméfaction & 4 & 28.6 \\
$(\mathrm{n}=14)$ & - & Nodule + mastodynia & 2 & 14 \\
& - & Other & 3 & 21.4 \\
Tracking anomaly $(\mathrm{n}=1)$ & & & 1 & - \\
\hline
\end{tabular}

All the patients underwent a breast ultrasound $(n=150)$ and 67 of the ultrasound mammography pair. On mammography, a mass was found in 41 patients, micro-calcifications in 18, density asymmetry in 12 and architectural distortion in 3 patients. In 8 cases, the mammogram returned to normal. On ultrasound, masses were the most common ultrasound abnormality $(n=122)$ in our sample. They were followed respectively by attenuating areas $(n=13)$, endocystic buds $(\mathrm{n}=10)$ and ductal dilations $(\mathrm{n}=5)$. After anatomopathological analysis of the breast lesions, ninety-eight (98) cases of benign lesions were found; sixteen (16) cases of intermediate lesions and thirty-six (36) cases of malignant lesions. The adenofibromas (ADF) were the most frequent benign tumors of the ACR 3 and ACR 4 classes followed by fibrocystic mastopathy (MFK) in the ACR 3 class and mastitis in the ACR 4 class. Infiltrating ductal carcinoma (ICC) was the most common histologic type among malignant lesions in all ACR classes. The distributions of the histological types of ACR lesions are given in the tables (Tables 4-6). 
Table 4. Distribution of ACR 3 lesions according to histological type $(n=60)$.

\begin{tabular}{cccc}
\hline Histological class & Histological types & Effective & Frequency (\%) \\
\hline ADF & MFK & 24 & 46 \\
& Phyllodes tumor & 11 & 21 \\
& Remodeled cyst & 5 & 9.6 \\
$\begin{array}{c}\text { Benign lesions } \\
(\mathrm{n}=52)\end{array}$ & Mastitis & 5 & 9.6 \\
& Abscess & 3 & 5.7 \\
& Galactocele & 1 & 1.9 \\
& Galactophoritis & 1 & 1.9 \\
& Apocrine metaplasia & 1 & 1.9 \\
$(\mathrm{n}=5)$ & HCA & 2 & 4.9 \\
Intermediate lesions & Sclorosing adenosis & 3 & 60 \\
$\begin{array}{c}\text { Malign lesions } \\
(\mathrm{n}=3)\end{array}$ & CCI or CINS & 2 & 67 \\
\hline
\end{tabular}

Table 5. Distribution of ACR 4 lesions according to histological type $(n=75)$.

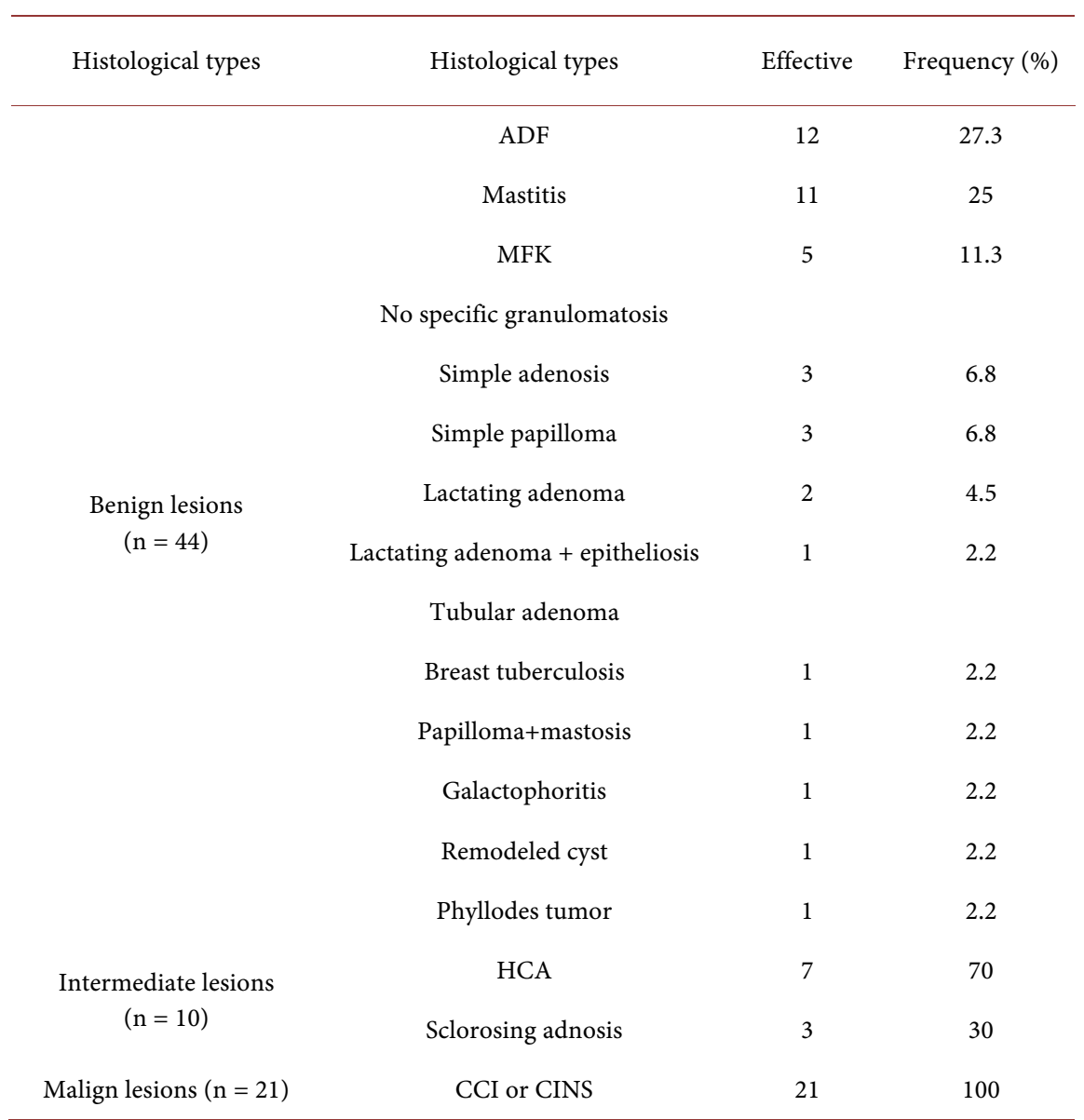


Table 6. Distribution of ACR 5 lesions according to histological type ( $\mathrm{n}=15)$.

\begin{tabular}{cccc}
\hline Histological class & Histological types & Effective & Frequency \\
\hline Benign lesions $(\mathrm{n}=2)$ & Mastitis & 2 & 100 \\
Intermediate lesions $(\mathrm{n}=1)$ & Sclorosing adenosis & 1 & 100 \\
& CCI or CINS & 11 & 91.7 \\
Maligne lesions $(\mathrm{n}=12)$ & Pleomorphic liposarcoma & 1 & 8.3 \\
\hline
\end{tabular}

\section{Discussion}

One hundred and fifty files had been retained for as many lesions biopsied under ultrasound guidance.

The average age of patients in general and during their menopause increased with the ACR class, so there is an association between age, late menopause and the ACR class. Breast cancer occurs at a younger age in our context contrary to the data in the literature [1] [4] [5] [6].

The presence or absence of familial breast cancer antecedent (ATCD) is not statistically related to the ACR classification $(\mathrm{p}=0.1)$ between the ACR 5 and non-ACR 5 groups. Non-exclusive breastfeeding and the state of nulliparity, on the other hand, are statistically linked to the ACR classification $(p=0.007)$ depending on whether the lesion is ACR 5 or not ACR 5.

Palpation of nodules was the most common clinical abnormality due to multiple national awareness efforts on breast self-palpation involving different social strata.

The adenofibromas were the benign lesions of young patients most often found in classes ACR 3 and ACR 4 because of their great benignity. They are generally classified as ACR 2 or ACR 3 requiring just ultrasound monitoring (Picture 1) but in certain circumstances they behave like malignant lesions classified as ACR 4 and whose histology alone will help in diagnostic confirmation. [4] [7].

MFKs with a polymorphic radiological presentation followed ADF in terms of frequency in the ACR 3 class. It constitutes a lesion group made up of fibrosis, adenosis and cyst most often classified ACR 3. When the cystic component is complex, it is classified ACR 4 [7].

Mastitis occurs at any age and is found in all ACR classes and particularly in ACR 4 class. It is above all the presence of associated signs such as:

- Intramammary or axillary lymph nodes and or lymphadenopathy.

- The state of infiltration and/or thickening of the skin which will guide their classification from one ACR to another.

Infiltrating ductal carcinoma ((CCI)) was the most frequent histological type of malignant tumors in all ACR classes, it constituted almost all of the ACR 4 malignant tumors (Picture 2). It most often occurred in older patients with obvious symptoms, especially in the ACR 4 and ACR 5 classes. Lesions found on mammography were also found on ultrasound. The associated signs were 

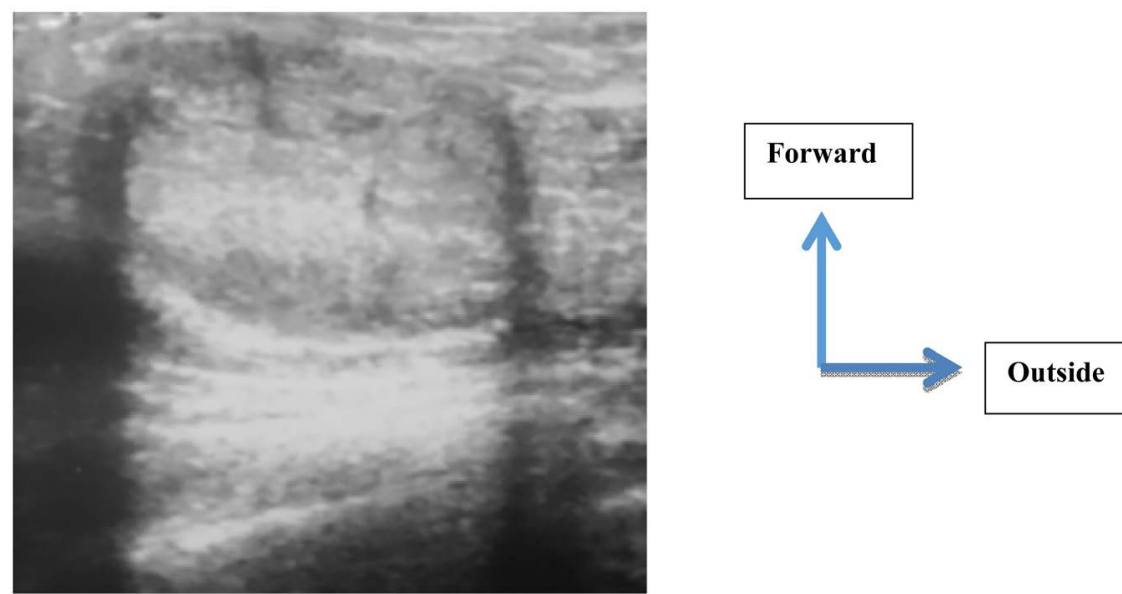

Picture 1. 19-year-old patient with no known pathological ATCD (menarche at 13 years), received for right mastodynia in whom the ultrasound notes a heterogeneous hyperechoic mass, with regular shape, with circumscribed contours, with a long axis parallel to the skin with side effects. She was classified ACR 3 and received an ultrasound guided biopsy. Pathologic result: adenofibroma (ADF).
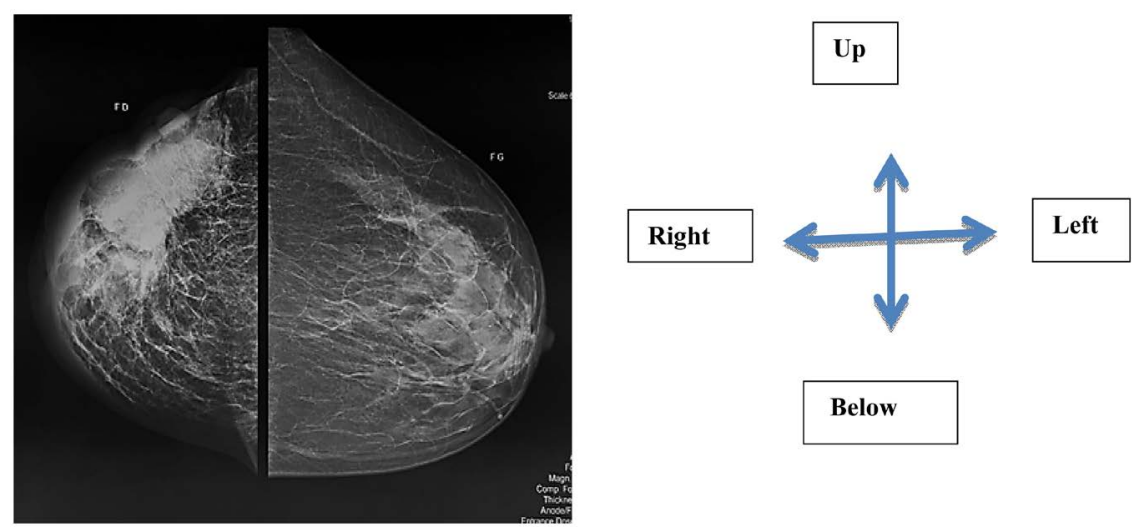

Picture 2. 70-year-old patient without familial ATCD of G10-P4 breast cancer, exclusive breastfeeding, menarche 17 years, without contraception or HRT received for a large fixed nodule. Mammography: microlobulated mass combining micro-calcifications of segmental distribution with architectural distortion. Ultrasound: heterogeneous hypoechoic mass presenting an attenuation of the echoes posteriorly, with axillary lymphadenopathy classified ACR 5. Pathological result: Invasive nonspecific carcinoma (SBR grade not specified).

suggestive and mainly concerned axillary lymphadenopathy and inflammatory thickening of the skin. The positive predictive value of breast cancer is $24 \%$ in the general population. It is high and is increasing compared to the $2015 \mathrm{WHO}$ data [1] [8] [9]. This finding can be explained by the genetic predispositions of black women in general. It can also be explained by the high genetic mutation rate (40\%) found in Ouédraogo in 2015 in his study of the histogenetic correlation of breast cancer in women $<40$ years old [9]. These malignant lesions were dominated by invasive ductal carcinomas, the SBR grade of which was $90 \%$ grade II. This means that the patient not only consults late but can also be explained by the aggressiveness of the cancers in our context [9] [10] [11]. 


\section{Conclusion}

Today breast cancer has become a real obsession, more so for the clinical practitioner than for the radiologist and pathologist. The aim of our study was to compare the radiological results with the pathological results and to deduce the positive predictive values. Cancerous lesions were found at an advanced stage (CCI) in all ACR classes (3-4-5). They were classified from one ACR to another not only by the radiological presentation of the lesions but also by the intervention of discriminatory elements such as age, family and personal ATCD. These discriminative elements are very important in senology, hence an interrogation and a systematic physical examination of the patient by the radiologist beforehand.

\section{Conflicts of Interest}

The authors declare no conflicts of interest regarding the publication of this paper.

\section{References}

[1] International Agency for Research on Cancer (2015) WHO: Latest Global Cancer Statistics. JAMA Oncology, 1, 505-527.

[2] Damoaliga, G. (2009) The Diagnostic and Therapeutic Difficulties of Breast Cancer in Women at the Yalgado Ouédraogo University Hospital in Ouagadougou: About 55 Cases. Doctoral Thesis $\mathrm{n}^{\circ} 106$ in Medicine, University of Ouagadougou UFR/SDS, 86 p.

[3] Nataf, A. (2016) Figures-Benchmarks-Measures-Classifications in Medical Imaging. Sauchamps Medical Montpellier France, 190 p.

[4] Benani, A. (2015) The Radiohistological Correlation of Subclinical Lesions of the Breast; about 32 Cases. Thesis $n^{\circ} 167$ of Doctorate in Medicine Year, Mohammed V Rabat University, 177 p.

[5] Ben Abdallah, M., Zehani, S., Hsairi, M., Hechinche, M., Ben Romdhane, K., et al. (2009) Breast Cancer in Tunisia: Epidemiological Characteristic and Trend of Incidence. Medical Tunisia, 87, 417-425.

[6] Boisseri-Lacroix, M. (2014) Radiohistological Correlation in Senology: Understanding Them in Order to Better Manage the Patient. Journal of Diagnostic and Interventional Radiology, 95, 125. https://doi.org/10.1016/j.diii.2014.02.001

[7] Stines, J. (2012) Breast Imaging. Medical Imaging Diagnosis, Elsevier Masson SAS: $423 \mathrm{p}$.

[8] National Cancer Institute (2017) Cancer Figures in France. http://www.e-cancer.fr/

[9] Marie Ouedraogo, N.L., Zongo, N., Zida, M., et al. (2018) Breat Cancer in Woman under 40 Years Old at Yalgado Ouedraogo University Hospital (Burkina Faso). JSM Surgical Oncology and Research, 3, 1022.

[10] Zoungrana, E.M.M. (2008) Histopathological Aspect of Breast Cancer Diagnosed in Ouagadougou; about 624 Cases Collected in 21 Years (1986-2006). Doctoral Thesis $\mathrm{n}^{\circ} 70$ in Medicine, $114 \mathrm{p}$.

[11] Lompo, G.M., Zoungrana, E.M.M., Zida, M., Lamien, A.M.S., Traoré, S.S. and Soudré, R.B. (2009) Histopathological Aspects of the Breast Cancers Diagnosed in Ouagadougou. African Journal of Cancer, No. 1, 207. 


\section{Abbreviations}

ACR: American College of Radiology; BIRADS: Breast Imaging Reporting And Data System; CHU: University Hospital Center; CHU-P: Pediatric University Hospital Center; CDD: Circumtance of discovery; CERS: Ethics Committee for Health Research; ADF: Adenofibroma; MKF: Fibrocystis Mastopathy; ICC: Infiltrating ductal carcinoma; CINS: not specified infiltrating carcinoma; HCA: Atypical ductal hyperplasia; ATCD: antecedents; Grade SBR: Histopronostic grade of Scarff-Bloom-Richardson.

\section{Annex: ACR classification}

ACR 0: Incomplete assessment (need for another review)

ACR 1: Negative examination; VPP $=0 \%$

ACR 2: benign findings: simple cysts, intramammary lymph node, stable post-surgical change; VPP $=0 \%$

ACR 3: probably benign anomaly: solid mass well circumscribed, parallel orientation, complicated cysts, clusters of PPV microcysts $<2 \%$

ACR 4: suspicious abnormality: a biopsy should be done PPV $=2 \%-95 \%$

ACR 5: high probability of malignancy. Imaging-guided biopsies will be able to confirm the diagnosis, especially in the perspective of a sentinel node technique or neo-adjuvant chemotherapy PPV > 95\%

ACR 6: the malignancy is known, proven by a biopsy, used during an extension or pre-treatment assessment, PPV 100\% 